\title{
Global Attractor for a Non-Autonomous Beam Equation*
}

\author{
Yonghua Ren ${ }^{1}$, Jianwen Zhang ${ }^{2 \#}$ \\ ${ }^{1}$ Department of Mathematic, Taiyuan University of Technology, Taiyuan, China \\ ${ }^{2}$ CSIRO Sustainable Ecosystems, Highett, Australia \\ Email: renyonghua@tyut.edu.cn, "jianwen.z2008@163.com
}

Received April 10, 2012; revised May 13, 2012; accepted May 21, 2012

\begin{abstract}
This work studies the global attractor for the process generated by a non-autonomous beam equation $u_{t t}+\Delta^{2} u+\eta u_{t}-\left[\beta(t)+M\left(\int_{\Omega}|\nabla u(x, t)|^{2} \mathrm{~d} x\right)\right] \Delta u+g(u, t)=f(x, t)$

Based on a time-uniform priori estimate method, we first in the space $H_{0}^{2}(\Omega) \times L^{2}(\Omega)$ establish a time-uniform priori estimate of the solution $u$ to the equation, and conclude the existence of bounded absorbing set. When the external term $f(x, t)$ is time-periodic, the continuous semigroup of solution is proved to possess a global attractor.
\end{abstract}

Keywords: Global Attractor; Non-Autonomous Beam Equation; Absorbing Set

\section{Introduction}

Let $\Omega$ be an open bounded connected domain in $R^{N}$ with smooth boundary $\partial \Omega$. In this work, we are devoted to the investigation of the following problem for a perturbed non-autonomous beam equation

$$
\begin{aligned}
& u_{t t}+\Delta^{2} u+\eta u_{t}-\left[\beta(t)+M\left(\int_{\Omega}|\nabla u(x, t)|^{2} \mathrm{~d} x\right)\right] \Delta u \\
& +g(u, t)=f(x, t), \quad x \in \Omega, t>\tau
\end{aligned}
$$

with the following initial and boundary conditions

$$
\begin{gathered}
\left.u\right|_{x \in \partial \Omega}=\left.\Delta u\right|_{x \in \partial \Omega}=0, \quad t \geq \tau \\
u(x, \tau)=u_{0 \tau}(x), u_{t}(x, \tau)=u_{1 \tau}(x), \quad x \in \Omega
\end{gathered}
$$

where $u(x, t)$ denotes a real-valued unknown function, and describes the transversal motion of the non-autonomous beam. $\eta \geq 0, \quad g \in C^{2}(R \times R ; R)$,

$f \in L^{\infty}\left(R ; L^{2}(\Omega)\right)$,

$$
f^{\prime}(\cdot, t) \in C_{b}\left(R ; L^{2}(\Omega)\right)=C\left(R ; L^{2}(\Omega)\right) \cap L^{\infty}\left(R ; L^{2}(\Omega)\right),
$$

and $\beta(t), g(u, t), \quad f(x, t)$ is the time-periodic external force.

System (1)-(3) is derived from the vibrations of an non-autonomous beam equation, and its dynamical set-

\footnotetext{
"This work is supported by the School Foundation of TUT (90010303020715) and the Provincial Natural Science Foundation of Shanxi (2010011008) and (2011021002-2).

${ }^{\#}$ Corresponding author.
}

ting is presented by Woinowsky-Krieger [1] as the new idea in fields of J. Appl. Mech. For the non-autonomous wave equations, there are many interesting results were published focusing different respects (see [2,3]). The focus of this work is the study of the long-term properties of the dynamical system generated by global attractor, please refer the reader to $[4,5]$ and references therein.

This paper is organized as follows. In Section 2, we introduce the main assumptions and discuss the existence of a family of solution operators $S(t, \tau)$ to the problem (1)-(3). Section 3 is devoted to the existence of the bounded absorbing set. In Section 4, we show the continuity of the semigroup operator. Finally, in Section 5, the global attractor is obtained.

\section{Assumptions and the Existence of the Solution Operators $S(t, \tau)$}

Firstly, we assume that the nonlinear functions $M, g$ satisfied the following conditions. Namely, there exist two constants $C_{1}, C_{2}>0$, such that

$$
\begin{aligned}
& \text { (H1) } \liminf _{|s| \rightarrow+\infty} \frac{G(s, t)}{s^{2}} \geq 0, G(s, t)=\int_{0}^{s} g(s, t) \mathrm{d} s \\
& \text { (H2) } \liminf _{|s| \rightarrow+\infty} \frac{s g(s, t)-C_{1} G(s, t)}{s^{2}} \geq 0 \\
& \text { (H3) } G_{t}^{\prime}(u, t) \leq \gamma G(u, t)+C_{2}, \gamma \leq \frac{\varepsilon C_{1}}{2}
\end{aligned}
$$


(H4) $\quad M^{\prime}(s) \geq 0, M(z) z \geq \tilde{M}(z)$

For simplicity, we define

$$
\phi(u, t)=\int_{\Omega} G(u(x), t) \mathrm{d} x, \tilde{M}(z)=\int_{0}^{z} M(s) \mathrm{d} s,
$$

and

$$
z(t)=|\nabla u(t)|^{2}
$$

with the usual notation, we write $H=L^{2}(\Omega)$,

$V=H_{0}^{2}(\Omega), \quad E=H_{0}^{2}(\Omega) \times L^{2}(\Omega)$ and the scalar products and norms on $H, V$ and $E$, respectively

$$
\begin{gathered}
(u, v)=\int_{\Omega} u v \mathrm{~d} x,|u|^{2}=(u, u), \forall u, v \in L^{2}(\Omega) \\
((u, v))=\int_{\Omega} \Delta u \Delta v \mathrm{~d} x,\|u\|^{2}=((u, u)), \forall u, v \in H_{0}^{2}(\Omega) \\
\forall \varphi_{i}=\left(u_{i}, v_{i}\right)^{T}, i=1,2 \\
\left(\varphi_{1}, \varphi_{2}\right)_{E}=\left(\left(u_{1}, u_{2}\right)\right)+\left(v_{1}, v_{2}\right),|\varphi|_{E}^{2}=(\varphi, \varphi)_{E}, \\
\varphi=(u, v)^{T} \in E
\end{gathered}
$$

For the linear self-adjoint and positive operator $A$, let us write $A=\Delta^{2}, A: D(A) \rightarrow H$.

The space $D(A)=\{v \in V, A v \in H\}$ is dense in $H$. Next, we define the power $A^{s}$ of $A, \forall s \in R$, which operate on the spaces $D\left(A^{s}\right)$, and write $V_{2 s}=D\left(A^{s}\right)$, which turns out to be a Hilbert space with the inner product and the norm

$$
(u, v)_{2 s}=\left(A^{s} u, A^{s} v\right),\|u\|_{2 s}^{2}=((u, u))_{2 s}, \forall u, v \in D\left(A^{s}\right)
$$

and $A^{\gamma}$ is an isomorphism from $D\left(A^{s}\right)$ onto $D\left(A^{s-\gamma}\right), \forall s, \gamma \in R$.

From the Poincaré inequality, there exists the constant $\lambda_{1}>0$, such that:

$$
|\Delta u| \geq \lambda_{1}|u|, \forall u \in V
$$

where $\lambda_{1}$ is the first-eigenvalue of $A^{\frac{1}{2}}$. For $\eta>0$, we consider the abstract Cauchy problem on $E$ in the unknown variables $u=u(\cdot, t)$

$$
\left\{\begin{array}{c}
u_{t t}+A u+\eta u_{t}+\left[\beta(t)+M\left(\int_{\Omega}\left|A^{\frac{1}{2}} u(x, t)\right|^{2} \mathrm{~d} x\right)\right] A^{\frac{1}{2}} u \\
+g(u, t)=f(x, t) \\
u(x, \tau)=u_{0 \tau}(x), \quad u_{t}(x, \tau)=u_{1 \tau}(x)
\end{array}\right.
$$

The following well-posedness result holds.

Theorem 1. Suppose that $\eta>0$ and the conditions (4)-(7) hold. If the nonlinear functions $g(u, t) \in C^{2}(R \times R ; R), \quad f(\cdot, t) \in L^{\infty}\left(R ; L^{2}(\Omega)\right)$. Then, for any initial value $\left(u_{0 \tau}, u_{1 \tau}\right)$ is given in $E$, problem (8) admits a unique solution $u=u(x, t)$ in the class

$$
\left(u(t), u_{t}(t)\right) \in C\left(R_{\tau} ; V\right) \times C\left(R_{\tau} ; H\right)
$$

where $R_{\tau}=[\tau,+\infty)$.

Furthermore, calling $\varphi(t)$ the difference of any two solutions corresponding to initial data having norm less than or equal to $\tau \geq 0$, there exists $C=C(\tau) \geq 0$ such that

$$
\varphi\left(t, \varphi_{0 \tau}\right)=e^{C(t-\tau)} \varphi_{0 \tau}+\int_{\tau}^{t} e^{C(t-s)} F(\varphi(s), s) \mathrm{d} s
$$

We omit the proof, based on a standard FeadoGalerkin approximation procedure together with a slight generalization of the usual Gronwall's lemma. Theorem 1 translates into the existence of the solution operators $S(t, \tau): E \rightarrow E, \quad t \geq \tau$ acting as

$$
\varphi_{0 \tau}=\left\{u_{0 \tau}, u_{1 \tau}\right\} \mapsto S(t, \tau) \varphi_{0 \tau}=\varphi(t)=\left(u(t), u_{t}(t)\right)
$$

Remark 1. In the non-autonomous case, namely, when both $\beta(t), \quad g(u, t)$ and $f(x, t)$ are time-dependent, the two-parameter family $S(t, \tau)$ fulfills the semigroup property

$$
S(t+s)=S(t)+S(s), \forall t, s \geq 0 .
$$

$S(\tau, \tau)=I$ is the identity operator, $\tau \geq 0$.

Thus, $S(t, \tau)$ is a continuous semigroup of operators on $E$.

\section{The Absorbing Set}

In this section, we prove the existence of an absorbing set for the semigroup $S(t, \tau)$. Combining with (4) and (5), there exist two constants $K_{1}, K_{2}>0$ such that

$$
\begin{gathered}
\varphi(u, t)+\frac{1}{8}\|u\|^{2} \geq-K_{1} \\
\int_{\Omega} u g(u, t) \mathrm{d} x-C_{1} \varphi(u, t)+\frac{1}{4}\|u\|^{2} \geq-K_{2}
\end{gathered}
$$

Lemma 1. Under the hypotheses of Theorem 1, For the ball $B=B(0, M), \forall\left(u_{0 \tau}, u_{1 \tau}\right) \in E$, centered at 0 of radius $M$, is an absorbing set for the semigroup $S(t, \tau)$ in $E$.

Proof. Let us begin with $\eta>0$ be fixed and $\varepsilon$ is chosen such that $0<\varepsilon<\min \left\{\frac{\eta}{4}, \frac{\lambda_{1}^{2}}{2 \eta}\right\}$. We set $v=u_{t}+\varepsilon u$ and rewrite (1) as follows

$$
\begin{aligned}
& v_{t}+(\eta-\varepsilon) v+\Delta^{2} u-\left[\beta(t)+M\left(\int_{\Omega}|\nabla u|^{2} \mathrm{~d} x\right)\right] \Delta u \\
& -\varepsilon(\eta-\varepsilon) u+g(u, t)=f(x, t)
\end{aligned}
$$

Taking the scalar product in $H$ of (12) with $v$, and we obtain the desired form 


$$
\begin{aligned}
& \frac{1}{2} \frac{\mathrm{d}}{\mathrm{d} t}\left[|v|^{2}+|\Delta u|^{2}+\beta(t)|\nabla u|^{2}+\tilde{M}(z)\right]+(\eta-\varepsilon)|v|^{2} \\
& +\varepsilon|\Delta u|^{2}-\varepsilon(\eta-\varepsilon)(u, v)+\varepsilon \tilde{M}(z)+(g(u, t), v) \\
& +\left(\varepsilon \beta(t)-\frac{\dot{\beta}(t)}{2}\right)|\nabla u|^{2} \leq(f(x, t), v)
\end{aligned}
$$

Using the Hölder inequality and the Poincaré inequality, we have the estimate

$$
\begin{aligned}
& (\eta-\varepsilon)|v|^{2}+\varepsilon|\Delta u|^{2}-\varepsilon(\eta-\varepsilon)(u, v) \\
& \geq \frac{3 \eta}{4}|v|^{2}+\varepsilon|\Delta u|^{2}-\frac{\varepsilon(\eta-\varepsilon)}{\sqrt{\lambda_{1}}}|\Delta u||v| \\
& \geq \frac{3 \eta}{4}|v|^{2}+\varepsilon|\Delta u|^{2}-\frac{\varepsilon}{2}|\Delta u|^{2}-\frac{\varepsilon \eta^{2}}{2 \lambda_{1}}|v|^{2} \\
& \geq \frac{\varepsilon}{2}|\Delta u|^{2}+\frac{\eta}{2}|v|^{2}
\end{aligned}
$$

Exploiting (11), we lead to

$$
\begin{aligned}
& (g(u, t), v) \\
& =\left(g(u, t), u_{t}\right)+\varepsilon(g(u, t), u) \\
& =\frac{\mathrm{d}}{\mathrm{d} t} \phi(u, t)-G_{t}^{\prime}(u, t)+\varepsilon(g(u, t), u) \\
& \geq \frac{\mathrm{d}}{\mathrm{d} t}[G(u, t)]+\frac{\varepsilon C_{1}}{2} G(u, t)-\frac{\varepsilon}{4}|\Delta u|^{2}-\varepsilon K_{2}-|\Omega| C_{2}
\end{aligned}
$$

and,

$$
(f, v) \leq \frac{|f|^{2}}{\eta}+\frac{\eta}{4}|v|^{2}
$$

where $|f|=\sup _{t \in R}|f(x, t)|$. From (13)-(16), it follows that

$$
\begin{aligned}
& \frac{1}{2} \frac{\mathrm{d}}{\mathrm{d} t}\left[|v|^{2}+|\Delta u|^{2}+\beta(t)|\nabla u|^{2}+\tilde{M}(z)+2 G(u, t)\right] \\
& +\left(\varepsilon \beta(t)-\frac{\dot{\beta}(t)}{2}\right)|\nabla u|^{2}+\varepsilon \tilde{M}(z)+\frac{\varepsilon C_{1}}{2} G(u, t) \\
& +\frac{\eta}{4}|v|^{2}+\frac{\varepsilon}{4}|\Delta u|^{2} \leq \varepsilon K_{2}+|\Omega| C_{2}+\frac{|f|^{2}}{\eta}
\end{aligned}
$$

So, in the light of condition (10), we have

$$
\begin{aligned}
L(t)= & |v|^{2}+|\Delta u|^{2}+\beta(t)|\nabla u|^{2}+\tilde{M}(z) \\
& +2 G(u, t)+2 K_{1}>0
\end{aligned}
$$

Taking $\theta=\min \left\{\frac{\varepsilon}{2}, \frac{\varepsilon C_{1}}{2},\left(2 \varepsilon-\frac{\dot{\beta}(t)}{\beta(t)}\right)\right\}$, we obtain

$$
\frac{\mathrm{d}}{\mathrm{d} t} L(t)+\theta L(t) \leq C
$$

where $C=2 \theta K_{1}+2 \varepsilon K_{2}+2|\Omega| C_{2}+\frac{2|f|^{2}}{\eta}$.

Applying the Gronwall's Lemma, we obtain the following absorbing inequality

$$
\begin{aligned}
|\varphi(t)|_{E}^{2} & \leq L(t) \\
& \leq L(\tau) \exp (-\theta(t-\tau))+\frac{C}{\theta}(1-\exp (-\theta(t-\tau)))
\end{aligned}
$$

or

$$
\limsup _{t \rightarrow+\infty}|\varphi(t)|_{E}^{2} \leq M, t \geq \tau
$$

Taking

$$
M=L(\tau) \exp (-\theta(t-\tau))+\frac{C}{\theta}(1-\exp (-\theta(t-\tau))) .
$$

Obviously, $|\varphi(t)|_{E}^{2} \leq M$. Furthermore, let us denote $B$ be a bounded closed ball of $E$ centered at 0 with radius $M$

$$
\begin{aligned}
B & =B_{E}(0, M) \\
& =\left\{(u, v) \in E:|\varphi(t)|_{E}^{2}=\|u(t)\|^{2}+|v(t)|^{2} \leq M\right\}
\end{aligned}
$$

So, $B$ is a bounded absorbing set of analytic semigroup $\{S(t, \tau)\}$ of (1)-(3). We complete the proof.

\section{Continuity of the Semigroup}

In this section, we prove the continuity of the semigroup $S(t, \tau)$ in $E$. For this reason, we assume

$$
\limsup _{|s| \rightarrow+\infty} \frac{\left|g^{\prime}(s, t)\right|}{|s|^{2}}=0, \forall 0 \leq \gamma_{0}<+\infty, \quad s \in R
$$

Lemma 2. Under the hypotheses of Theorem 1, the mapping $\left\{u_{0 \tau}, u_{1 \tau}\right\} \rightarrow\left\{u(t), u_{t}(t)\right\}$, for $\forall\left(u_{0 \tau}, u_{1 \tau}\right) \in E$, is continuous in $E$, and the semigroup $S(t, \tau)$ associated with the initial-boundary value problem (1) is a $C_{0}$-semigroup in $E$.

Proof. Assume that $B \subset E$ be a bounded positive invariant set for $S(t, \tau)$, and initial-data

$\left\{u_{0 \tau}, u_{1 \tau}\right\},\left\{v_{0 \tau}, v_{1 \tau}\right\} \in E$. Let $u, v$ be the two corresponding solutions of (1).

Assume $\xi=u-v$. We claim that the proof is similar to the Lemma 1. Then, from (1) we have

$$
\begin{aligned}
& \xi_{t t}+\Delta^{2} \xi-\beta(t) \Delta \xi+\eta \xi \\
& -M\left(\int_{\Omega}|\nabla u(x, t)|^{2} \mathrm{~d} x\right) \Delta u \\
& +M\left(\int_{\Omega}|\nabla v(x, t)|^{2} \mathrm{~d} x\right) \Delta v \\
& +(g(u, t)-g(v, t))=f_{1}(x, t)-f_{2}(x, t)
\end{aligned}
$$

By multiplying (21) by $w=\xi_{t}+\varepsilon \xi$ and integrating 
over $\Omega$, we find

$$
\begin{aligned}
& \frac{1}{2} \frac{\mathrm{d}}{\mathrm{d} t}\left[|w|^{2}+|\Delta \xi|^{2}\right]+(\eta-\varepsilon)|w|^{2}+\varepsilon|\Delta \xi|^{2} \\
& -\varepsilon(\eta-\varepsilon)(\xi, w)-\beta(t)(\Delta \xi, w) \\
& \left(-M\left(\int_{\Omega}|\nabla u(x, t)|^{2} \mathrm{~d} x\right) \Delta u+M\left(\int_{\Omega}|\nabla v(x, t)|^{2} \mathrm{~d} x\right) \Delta v, w\right) \\
& +(g(u, t)-g(v, t), w)=\left(f_{1}(x, t)-f_{2}(x, t), w\right)
\end{aligned}
$$

Next, we are devoted to estimate (22). By the same method that we obtained (14), it follows that

$$
\begin{aligned}
& (\eta-\varepsilon)|w|^{2}+\varepsilon|\Delta \xi|^{2}-\varepsilon(\eta-\varepsilon)(\xi, w) \\
& \geq \frac{\eta}{2}|w|^{2}+\frac{\varepsilon}{2}|\Delta \xi|^{2}
\end{aligned}
$$

And, according to (20), for $\forall \varepsilon>0$, there exists a constant $C_{\varepsilon}>0$, such that

$$
\left|g^{\prime}(s, t)\right| \leq \varepsilon|s| \gamma_{0}+C_{\varepsilon}, \quad \forall 0 \leq \gamma_{0}<+\infty
$$

By (24) and the Sobolev embedding theorem, we can obtain $g(u, t), g^{\prime}(u, t)$ are uniformly bounded in $L^{\infty}$, that is, there exists a constant $K_{3}>0$, such that

$$
|g(u, t)|_{L^{\infty}} \leq K_{3},\left|g^{\prime}(u, t)\right|_{L^{\infty}} \leq K_{3}
$$

From (24), (25), it follows that

$$
\begin{aligned}
& (g(u, t)-g(v, t), w)=\int_{\Omega} g^{\prime}(u+\vartheta \xi) \cdot \xi \cdot w \mathrm{~d} x, \\
& \vartheta=\vartheta(x) \in[0,1]
\end{aligned}
$$

Thus

$$
\begin{aligned}
& |(g(u, t)-g(v, t), w)| \\
& =\left|\int_{\Omega} g^{\prime}(u+\vartheta \xi) \cdot \xi \cdot w \mathrm{~d} x\right| \\
& \geq-K_{3} \int_{\Omega}|\xi| \cdot|w| \mathrm{d} x \geq-\frac{K_{3}}{\lambda_{1}} \int_{\Omega}|\Delta \xi| \cdot|w| \mathrm{d} x
\end{aligned}
$$

Meanwhile, we know easily

$$
\begin{aligned}
& \mid \beta(t)(\Delta(\xi, w) \mid \\
& \leq \beta(t)|\Delta \xi||w| \leq \frac{C_{3}}{2}\left(|\Delta \xi|^{2}+|w|^{2}\right)
\end{aligned}
$$

Due to the continuity of $M^{\prime}$, we can note the fact that

$$
\begin{aligned}
& M\left(x^{2}\right)-M\left(y^{2}\right) \\
& \leq M^{\prime}\left(\sup \left\{x^{2}, y^{2}\right\}\right)|x+y| \cdot|x-y| .
\end{aligned}
$$

We see that easily

$$
\begin{aligned}
& \left(M\left(\int_{\Omega}|\nabla u|^{2} \mathrm{~d} x\right) \Delta u-M\left(\int_{\Omega}|\nabla v|^{2} \mathrm{~d} x\right) \Delta v, w\right) . \\
& \leq C_{4}|\Delta \xi||w|
\end{aligned}
$$

This implies

$$
\begin{aligned}
& \mid\left(M\left(\int_{\Omega}|\nabla u|^{2} \mathrm{~d} x\right) \Delta u-M\left(\int_{\Omega}|\nabla v|^{2} \mathrm{~d} x\right) \Delta v, w \mid\right. \\
& =\mid M\left(\int_{\Omega}|\nabla u|^{2} \mathrm{~d} x\right) \Delta u-M\left(\int_{\Omega}|\nabla u|^{2} \mathrm{~d} x\right) \Delta v \\
& \quad+M\left(\int_{\Omega}|\nabla u|^{2} \mathrm{~d} x\right) \Delta v-M\left(\int_{\Omega}|\nabla v|^{2} \mathrm{~d} x\right) \Delta v, w \mid \\
& =\mid M\left(\int_{\Omega}|\nabla u|^{2} \mathrm{~d} x\right) \Delta \xi \\
& \quad+\left(M\left(\int_{\Omega}|\nabla u|^{2} \mathrm{~d} x\right)-M\left(\int_{\Omega}|\nabla v|^{2} \mathrm{~d} x\right)\right) \Delta v, w \mid \\
& \leq C_{5}|\Delta \xi| \cdot|w|+C_{4}|\Delta \xi| \cdot|w| \\
& \leq \frac{C_{6}}{2}\left(|\Delta \xi|^{2}+|w|^{2}\right)
\end{aligned}
$$

where $C_{6}=\max \left\{C_{4}, C_{5}\right\}$.

Combining with (27) and (28), it is obtained directly

$$
\begin{aligned}
& |\beta(t)(\Delta \xi, w)| \\
& +\left(M\left(\int_{\Omega}|\nabla u|^{2} \mathrm{~d} x\right) \Delta u-M\left(\int_{\Omega}|\nabla v|^{2} \mathrm{~d} x\right) \Delta v, w\right) \\
& \leq \frac{1}{2}\left(C_{3}+C_{6}\right)\left(|\Delta \xi|^{2}+|w|^{2}\right) \leq C_{7}\left(|\Delta \xi|^{2}+|w|^{2}\right)
\end{aligned}
$$

Thanks to (22)-(29), and the usual Gronwall's Lemma, we have

$$
|\Delta \xi(t)|^{2}+|w(t)|^{2} \rightarrow 0, \text { as }|\Delta \xi(\tau)|^{2}+|w(\tau)|^{2} \rightarrow 0 .
$$

So we complete the proof.

\section{Existence of the Global Attractor}

Theorem 2. Under the hypotheses of Theorem 1, the semigroup $S(t, \tau)$ associated with the initial-boundary value problem (1) possesses a global attractor $\beta$ in $E$ which attracts all bounded subsets of $E$.

Proof. Let $\left(u(t), u_{t}(t)\right)$ be a solution of (12) with initial value, for any $\left(u_{0 \tau}, u_{1 \tau}\right) \in E$, and, it can be decomposed into $\left(u, u_{t}\right)=\left(u_{1}, u_{1 t}\right)+\left(u_{2}, u_{2 t}\right)$, where $v_{i}(t)=u_{i t}(t)+\varepsilon u_{i}(t), \quad i=1,2$, satisfy, respectively,

$$
\left\{\begin{array}{l}
v_{t}+(\eta-\varepsilon) v+\Delta^{2} u-\varepsilon(\eta-\varepsilon) u=0, \quad t \geq \tau \\
u_{1}(\tau)=u_{0 \tau}, \quad u_{1 t}(\tau)=u_{1 \tau}
\end{array}\right.
$$

and

$$
\left\{\begin{array}{l}
v_{t}+(\eta-\varepsilon) v+\Delta^{2} u-\varepsilon(\eta-\varepsilon) u=b(t), \quad t \geq \tau \\
u_{2}(\tau)=0, \quad u_{2 t}(\tau)=0, \\
b(t)=\left[\beta(t)+M\left(\int_{\Omega}|\nabla u|^{2} \mathrm{~d} x\right)\right] \Delta u-g(u, t)+f(x, t)
\end{array}\right.
$$

Applying two lemmas showed below. We also define a new inner product and norm in $E=H_{0}^{2}(\Omega) \times L^{2}(\Omega)$. Obviously, by embedding theorem, (30) are easily con- 
cluded through some simple computation $\left|v_{1}(t)\right|_{E}^{2} \leq C_{8}$. It is sufficient to prove that $S(t, \tau)$ is asymptotically smooth in $E$.

Taking the inner product in $E$ of (12) with $v_{2}=u_{2 t}+\varepsilon u_{2}$, we obtain

$$
\begin{aligned}
& \frac{1}{2} \frac{\mathrm{d}}{\mathrm{d} t}\left[\left|v_{2}\right|^{2}+\left|\Delta u_{2}\right|^{2}+\beta(t)\left|\nabla u_{2}\right|^{2}+\tilde{M}(z)\right]+(\eta-\varepsilon)\left|v_{2}\right|^{2} \\
& +\varepsilon\left|\Delta u_{2}\right|^{2}-\varepsilon(\eta-\varepsilon)\left(u_{2}, v_{2}\right)+\varepsilon \tilde{M}(z)+\left(g(u, t), v_{2}\right) \\
& +\left(\varepsilon \beta(t)-\frac{\dot{\beta}(t)}{2}\right)\left|\nabla u_{2}\right|^{2} \leq\left(f(x, t), v_{2}\right)
\end{aligned}
$$

According to the uniform boundedness of $u$ in space $V$, the Sobolev Embedding theorem and the Gromwall's Lemma, combining with Lemma 1, we have the conclusion similar to below, $\left|v_{2}(t)\right|_{E}^{2} \leq C_{9}$. Thus, this leads to $S(t, \tau)$ possesses a global attractor $\beta$ in $E$ which attracts all bounded subsets of $E$. So we end the proof.

\section{REFERENCES}

[1] S. Woinowsky-Krieger, "The Effect of Axial Force on the Vibration of Hinged Bars,” Journal of Applied Mechanics, Vol. 17, 1950, pp. 35-36.

[2] O. F. Ma, S. H. Wang and C. K. Zhong, "Necessary and Sufficient Conditions for the Existence of Global Attractor for Semigroup and Application,” Indiana University Mathematics Journal, Vol. 51, 2002, pp. 529-551. doi.org/10.1512/iumj.2002.51.2255

[3] R. Temam, "Infinite Dimensional Dynamical System in Mechanics and Physical,” 2nd Edition, Spring-Verlag, Nork York, 1997.

[4] Q. Z. Ma and C. K. Zhong, "Existence of Strong Global Attractors for Hyperbolic Equation with Linear Memory," Applied Mathematics and Computation, Vol. 157, No. 1, 2004, pp. 745-758. doi:10.1016/j.amc.2003.08.080

[5] Q. Z. Ma and C. K. Zhong, "Global Attractors of Strong Solutions for Nonclassical Diffusion Equation,” Journal of Lanzhou University, Vol. 40, 2004, pp. 7-9. 